\title{
An Expected Least-Squares Beamforming Approach to Signal Estimation With Steering Vector Uncertainties
}

\author{
Yonina C. Eldar, Member, IEEE, Arye Nehorai, Fellow, IEEE, and Patricio S. La Rosa
}

\begin{abstract}
We treat the problem of beamforming for signal estimation in the presence of steering vector uncertainties, where the goal is to estimate a signal amplitude from a set of array observations. Conventional beamforming methods typically aim at maximizing the signal-to-interference-plus-noise ratio (SINR). Recently, a maximum likelihood (ML) approach was introduced that leads to an iterative beamformer. Here we suggest an expected least-squares (LS) strategy that results in a simple linear beamformer. We then demonstrate through simulations that the LS beamformer often performs similarly to the ML method in terms of mean-squared error and outperforms conventional SINR-based approaches.
\end{abstract}

Index Terms-Array processing, beamforming, least squares (LS), random steering vector, signal estimation.

\section{INTRODUCTION}

B EAMFORMING methods are used extensively in a variety of areas, where one of their goals is to estimate the source signal amplitude $s(t)$ from the array observations

$$
\mathbf{y}(t)=s(t) \mathbf{a}+\mathbf{i}(t)+\mathbf{e}(t), \quad 1 \leq t \leq N .
$$

Here $\mathbf{y}(t) \in \mathbb{C}^{M}$ is the complex vector of array observations at time $t$ with $M$ being the number of array sensors, $s(t)$ is the signal amplitude, $\mathbf{a}$ is the signal steering vector that depends on the direction of arrival (DOA) of the wavefront plane associated with $s(t), \mathbf{i}(t)$ is the interference, $\mathbf{e}(t)$ is a Gaussian noise vector, and $N$ is the number of snapshots [1]. We estimate the signal amplitude $s(t)$ from the observations $\mathbf{y}(t)$ using a set of beamformer weights $\mathbf{w}(t)$, where the output of a narrowband beamformer is given by

$$
\hat{s}(t)=\mathbf{w}^{*}(t) \mathbf{y}(t), \quad 1 \leq t \leq N .
$$

In some applications, such as in the case of a fully calibrated array, the steering vector can be assumed to be known exactly.

Manuscript received July 5, 2005; revised October 12, 2005. The work of Y. Eldar was supported in part by the Israel Science Foundation. The work of A. Nehorai and P. S. La Rosa was supported in part by the Air Force Office of Scientific Research under Grant FA9550-05-1-0018 and in part by the National Science Foundation under Grants CCR-0105334 and CCR-0330342. The associate editor coordinating the review of this manuscript and approving it for publication was Dr. Stefano Buzzi.

Y. C. Eldar is with the Department of Electrical Engineering, Technion-Israel Institute of Technology, Haifa 32000, Israel (e-mail: yonina@ee.technion.ac.il).

A. Nehorai and P. S. La Rosa are with the Department of Electrical and Systems Engineering, Washington University in St. Louis, St. Louis, MO 63130 USA.

Digital Object Identifier 10.1109/LSP.2006.870356
However, in practice, the array response may have some uncertainties or perturbations in the steering vector, due to errors in sensor positions, gains or phases, mutual couplings between sensors, receiver fluctuations due to temperature changes, quantization effects, and coherent and incoherent local scatters [2], [3]. One approach to account for these uncertainties is to model their effects [4]. However, these perturbations often take place simultaneously, which significantly complicates the model. Instead, the uncertainties in a can be taken into account by treating it as a deterministic vector that lies in an ellipsoid centered at a nominal steering vector [3], [5]. An alternative strategy has been to treat the steering vector as a random vector assuming knowledge of its distribution [6] or the second-order statistics [7]-[11]. In the latter case, the mean value of a corresponds to the nominal steering vector, and the covariance matrix captures its perturbations.

Here we treat the case in which $\mathbf{a}$ is modeled as a random vector with known mean $\mathbf{m}$ and covariance matrix $\mathbf{C}$. The typical beamforming approach in this setting is to maximize the signal-to-interference-plus-noise ratio (SINR), resulting in the principal eigenvector beamformer [12]. Despite the fact that the SINR has been used as a measure of beamforming performance and as a design criterion in many beamforming approaches, we note that maximizing SINR may not guarantee a good estimate of the signal. In an estimation context, where our goal is to design a beamformer in order to obtain an estimate of the signal amplitude that is close to its true value, it is more informative to consider the estimation error as a measure of performance.

In [11], a maximum-likelihood (ML) approach was suggested in which the steering vector is chosen to maximize the observations' likelihood. The solution assumes that the steering vector is complex Gaussian with known mean and covariance, and the noise is given by a zero-mean complex Gaussian vector with known covariance. Since the ML solution depends on $s(t)$, and therefore cannot be found explicitly, an iterative method was proposed; however, no proof of convergence was given. Furthermore, the suggested beamformer is not given in closed form and is computationally involved.

In this letter, we suggest a simple beamforming strategy resulting in a closed-form linear beamformer. Our method, which is also outlined in [13], is based on an expected least-squares (LS) estimator proposed in [14]. The resulting beamformer has a similar form to the recursive ML solution [11] but is explicit. As we show in the numerical examples, the LS beamformer often performs similarly to the ML approach in terms of mean-squared error (MSE) and outperforms the conventional and robust SINRbased methods, even when the same prior knowledge is used. 


\section{EXPECTED LS BEAMFORMING}

The most common approach to designing beamformers is to maximize the SINR. In the case of a random steering vector, this methods results in the principal eigenvector beamformer

$$
\mathbf{w}=\alpha \mathcal{P}\left\{\mathbf{R}^{-1} \mathbf{R}_{s}\right\}
$$

where $\mathcal{P}\{\mathbf{A}\}$ denotes the principal eigenvector of the matrix $\mathbf{A}$, $\alpha$ is chosen such that $\mathbf{w}^{*} \mathbf{R}_{s} \mathbf{w}=1$, and for brevity, we omitted the index $t$. Here

$$
\mathbf{R}=E\left\{(\mathbf{i}+\mathbf{n})(\mathbf{i}+\mathbf{n})^{*}\right\}
$$

is the interference + noise covariance matrix, which is typically replaced by an estimate such as the sample covariance matrix $\widehat{\mathbf{R}}_{s m}$ with diagonal loading, and

$$
\mathbf{R}_{s}=E\left\{\mathbf{a a}^{*}\right\}=\mathbf{C}+\mathbf{m m}^{*}
$$

is the signal correlation matrix.

Although maximizing the SINR is a popular design strategy, choosing $\mathbf{w}$ to maximize the SINR does not necessarily result in an estimated signal amplitude $\hat{s}$ that is close to $s$. Following the ideas in [14], we now consider the LS beamformer for the case of a random steering vector, in which we minimize the expected LS error.

For a fixed steering vector, maximizing the SINR is equivalent to minimizing the weighted LS error

$$
\epsilon_{\mathrm{LS}}=(\mathbf{y}-\mathbf{a} s) \mathbf{R}^{-1}(\mathbf{y}-\mathbf{a} s)
$$

which is the weighted data error, i.e., the error between the given and estimated observations. This relation no longer holds true when the steering vector is random. In this case, $\epsilon_{\mathrm{LS}}$ is a random variable and therefore cannot be minimized. Instead, we consider minimizing the expected LS error where the expectation is taken with respect to a

$$
\begin{aligned}
& E\left\{\epsilon_{\mathrm{LS}}\right\} \\
& =E\left\{(\mathbf{y}-\mathbf{m} s-(\mathbf{a}-\mathbf{m}) s)^{*} \mathbf{R}^{-1}(\mathbf{y}-\mathbf{m} s-(\mathbf{a}-\mathbf{m}) s)\right\} \\
& =(\mathbf{y}-\mathbf{m} s)^{*} \mathbf{R}^{-1}(\mathbf{y}-\mathbf{m} s)+s^{2} E\left\{(\mathbf{a}-\mathbf{m})^{*} \mathbf{R}^{-1}(\mathbf{a}-\mathbf{m})\right\} \\
& =(\mathbf{y}-\mathbf{m} s)^{*} \mathbf{R}^{-1}(\mathbf{y}-\mathbf{m} s)+s^{2} \operatorname{Tr}\left(\mathbf{R}^{-1} \mathbf{C}\right) .
\end{aligned}
$$

Differentiating (7) with respect to $\hat{s}$ and equating to 0 , we have

$$
\hat{s}=\frac{1}{\operatorname{Tr}\left(\mathbf{R}^{-1} \mathbf{C}\right)+\mathbf{m}^{*} \mathbf{R}^{-1} \mathbf{m}} \mathbf{m}^{*} \mathbf{R}^{-1} \mathbf{y} .
$$

Thus, the LS beamformer is

$$
\mathbf{w}_{\mathrm{LS}}=\frac{1}{\operatorname{Tr}\left(\mathbf{R}^{-1} \mathbf{C}\right)+\mathbf{m}^{*} \mathbf{R}^{-1} \mathbf{m}} \mathbf{R}^{-1} \mathbf{m} .
$$

We first note that unlike the case in which a is known, the LS approach is not equivalent to the max-SINR beamformer of (3). Interestingly, however, the method of (9) is a scaled version of the max-SINR beamformer when $\mathbf{a}=\mathbf{m}$. Thus, the effect of the uncertainty is to change the scaling of the beamformer. It is well known in the estimation literature that scaling an estimator can significantly reduce its MSE. For example, the class of James-Stein estimators [15]-[17] are scaled versions of the LS estimator that have provably smaller MSE.

In Section III, we illustrate, through numerical examples, that for a wide range of signal-to-noise-ratio (SNR) values, the LS beamformer has a smaller MSE than the principal eigenvector solution (3) and the robust solutions [3], [5], [10]. These observations are true, even when $\mathbf{C}$ is not known exactly, but is chosen in an ad hoc manner, and $\mathbf{R}$ is estimated by $\widehat{\mathbf{R}}_{s m}$ with an ad hoc diagonal loading. In particular, we choose $\mathbf{m}$ and $\mathbf{C}$ so that our model of the random steering vector is the same in average to the one used by the robust SINR-based method in [5]. Therefore, in terms of MSE, the LS approach appears to often be preferable over standard and robust methods while requiring the same prior knowledge.

\section{NUMERICAL EXAMPLES}

We now compare the performance of the LS beamformer with other techniques.

The examples below consist of a uniform linear array of $M=$ 10 omnidirectional sensors spaced half a wavelength apart. In all the examples, $s(t)$ is a complex sine wave with varying average-power $P_{s}$, chosen to obtain the desired average array SNR defined as [18]

$$
\mathrm{SNR}=10 \log _{10}\left(\frac{P_{s}\left(\mathbf{m}^{*} \mathbf{m}+\operatorname{Tr}(\mathbf{C})\right)}{\sigma_{e}^{2}}\right)
$$

where $\sigma_{e}^{2}$ is the noise power in each sensor. The noise e $(t)$ is a zero-mean, Gaussian, complex random vector, temporally and spatially white, with variance $\sigma_{e}^{2}=1$ in each sensor. The interference is given by $\mathbf{i}(t)=\mathbf{a}_{i} i(t)$, where $\mathbf{a}_{i}$ is the interference steering vector, and $i(t)$ is a zero-mean, Gaussian, complex temporally white process. We used an interference with $\mathrm{DOA}=-30^{\circ}$, interference-to-noise-ratio INR $=20 \mathrm{~dB}$, a number of training snapshots $N=30$. The signal is continuously present throughout the training data. As in [18], we consider two scenarios for the steering vector covariance matrix: 1) $\mathbf{C}$ is a diagonal matrix, where $\mathbf{a}$ is drawn from a Gaussian, complex random vector with mean $\mathbf{m}$ and covariance matrix $\mathbf{C}=\sigma_{a}^{2} \mathbf{I}$; and 2) $\mathbf{C}$ is a nondiagonal matrix, where $\mathbf{a}$ is drawn from a local scatter model given by $\mathbf{a}=\mathbf{m}+(1 / \sqrt{L}) \sum_{k=1}^{L} g_{k} \mathbf{a}\left(\theta_{k}\right)$, where $L=10$ is the number of scatterers, $\left\{g_{k}\right\}$ are zero-mean, independent, and identically distributed Gaussian random variables with variance $\sigma_{g}^{2}$, and $\left\{\theta_{k}\right\}$ are independent Gaussian random variables with mean $\theta_{0}$ and standard deviation (scatter angular spread) $\sigma_{\theta}=15^{\circ}$. The covariance matrix is given by $\mathbf{C}=\sigma_{g}^{2} \int_{-\pi}^{\pi} \mathbf{a}\left(\theta_{k}\right) \mathbf{a}^{*}\left(\theta_{k}\right) p(\theta) d \theta$, where $p(\theta)$ is the pdf of $\theta$ [18]. In both cases, the steering vector was independently drawn for each simulation run, $\mathbf{m}$ represents the nominal steering vector with DOA $\theta_{0}=50^{\circ}$, and $\sigma_{a}^{2}$ as well as $\sigma_{g}^{2}$ varied to obtain the desired uncertainty ratio (UR) defined by

$$
\mathrm{UR}=10 \log _{10}\left(\frac{\operatorname{Tr}(\mathbf{C})}{\mathbf{m}^{*} \mathbf{m}}\right) .
$$


TABLE I

BEAMFORMERS USED IN THE NUMERICAL EXAMPLES

\begin{tabular}{|c|c|}
\hline Beamformer & Expression \\
\hline PEIG & $\alpha_{1} \mathcal{P}\left\{\left(\widehat{\mathbf{R}}_{s m}+\lambda \mathbf{I}\right)^{-1} \mathbf{R}_{s}\right\}$ \\
ROB1 & $\frac{\alpha_{2}}{\sqrt{M}} \cdot \frac{\left(\delta \widehat{\mathbf{R}}_{s m}+\mathbf{I}\right)^{-1} \mathbf{m}}{\mathbf{m}^{*}\left(\delta \widehat{\mathbf{R}}_{s m}+\mathbf{I}\right)^{-1} \widehat{\mathbf{R}}_{s m}\left(\delta \widehat{\mathbf{R}}_{s m}+\mathbf{I}\right)^{-1} \mathbf{m}}$ \\
ML & $\frac{\alpha_{3} \mathcal{P}\left\{\left(\widehat{\mathbf{R}}_{s m}+\lambda \mathbf{I}\right)^{-1}\left(\mathbf{R}_{s}-\epsilon_{2} \mathbf{I}\right)\right\}}{\left(\hat{\mathbf{R}}_{s m}+\lambda \mathbf{I}\right)^{-1} \mathbf{a}_{p o s t}}$ \\
LS & $\frac{\left(\widehat{\mathbf{R}}_{s m}+\lambda \mathbf{I}\right)^{-1} \mathbf{m}}{\operatorname{Tr}\left\{\left(\hat{\mathbf{R}}_{s m}+\lambda \mathbf{I}\right)^{-1} \mathbf{C}_{p o s t}\right\}+\mathbf{m}_{p o s t}^{*}\left(\widehat{\mathbf{R}}_{s m}+\lambda \mathbf{I}\right)^{-1} \mathbf{a}_{p o s t}}$ \\
\hline
\end{tabular}

To evaluate the performance of the different methods, we used the square root of the normalized mean-square error (NMSE). For each experiment $k$, we computed the NMSE as

$$
\mathrm{NMSE}_{k}=\frac{\sum_{t=1}^{N}\left|\hat{s}_{k}(t)-s_{k}(t)\right|^{2}}{\sum_{t=1}^{N}\left|s_{k}(t)\right|^{2}}, \quad 1 \leq k \leq K
$$

where $s_{k}(t)$ and $\hat{s}_{k}(t)$ are the desired signal and its estimate for a given experiment $k$, and $K$ is the total number of experiments. Each result presented below was obtained by averaging $K=$ 500 Monte Carlo experiments.

We compared the performance of the LS beamformer to four other beamformers: the principal eigenvector (PEIG) beamformer [12], the robust beamformers (ROB1 and ROB2) [5], [10], and the ML beamformer [11]. Table I summarizes the beamformers implemented in the simulations. In the table, $\widehat{\mathbf{R}}_{s m}$ is the estimate of the sample covariance matrix; $\mathbf{R}_{s}$ is the signal correlation matrix (5); $\lambda$ is an ad hoc diagonal load; $\delta$ is chosen such that $\left\|\left(\mathbf{I}+\delta \widehat{\mathbf{R}}_{s m}\right)^{-1} \mathbf{m}\right\|=\epsilon_{1}$, with $\epsilon_{1}$ the uncertainty level on the square norm of the steering vector error [5]; $\mathbf{m}$ is the nominal steering vector; $\alpha_{1}$ is chosen so that the corresponding beamformer satisfies $\mathbf{w}^{*} \mathbf{R}_{s} \mathbf{w}=1, \alpha_{2}=\left\|\left(\widehat{\mathbf{R}}_{s m}^{-1}+\lambda \mathbf{I}\right)^{-1} \mathbf{m}\right\|^{2} ; \alpha_{3}$ is chosen so that $\mathbf{w}^{*}\left(\mathbf{R}_{s}-\epsilon_{2} \mathbf{I}\right) \mathbf{w}=1$ with $\epsilon_{2}$ the uncertainty level on the norm of the signal correlation matrix error [10]; and $\mathbf{a}_{\text {post }}$ and $\mathbf{C}_{\text {post }}$ are the a posteriori steering vector and signal covariance, respectively (see [11] for details on their recursive expressions).

We implemented the beamformers for a) known $\mathbf{R}, \mathbf{m}$, and $\mathbf{C}$; b) known $\mathbf{m}$ and $\mathbf{C}$ and estimated $\mathbf{R}$; and c) known $\mathbf{m}$ and estimated $\mathbf{R}$ and $\mathbf{C}$. The parameters of the compared methods were chosen according to each of the assumptions above. For example, under assumption a), we set $\lambda=0$, and $\widehat{\mathbf{R}}_{s m}$ is replaced by $\mathbf{R}$ for the PEIG, ROB1, ML, and LS beamformers. For scenario 2), the elements of $\mathbf{C}$ are obtained by numerically approximating the integral.ForROB 1 , we set $\epsilon_{1}=4$; forROB2, we set $\epsilon_{2}=0$ because $\mathbf{m}$ and $\mathbf{C}$ are known; therefore, its performance is the same as the PEIG beamformer. Under assumptions b) and c), we estimate $\mathbf{R}$ using a diagonal loading approach with $\lambda=30$. For the purpose of comparison with ROB1, assuming that there is no access to the true $\mathbf{C}$ matrix [assumption c)], we set $\mathbf{C}=\nu \mathbf{I}$, where $\nu$ is chosen (a)

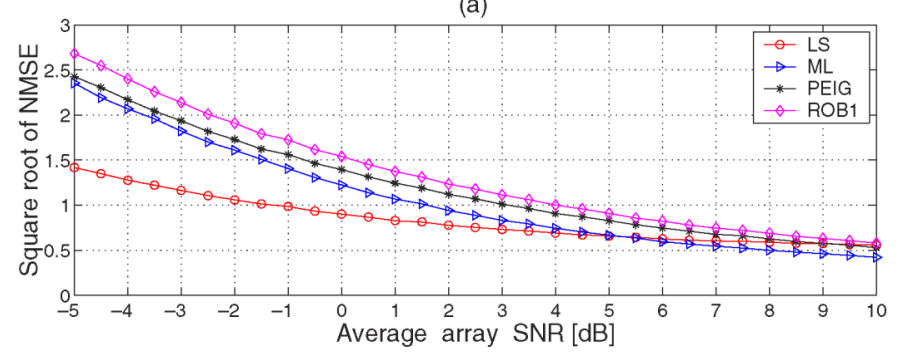

(b)

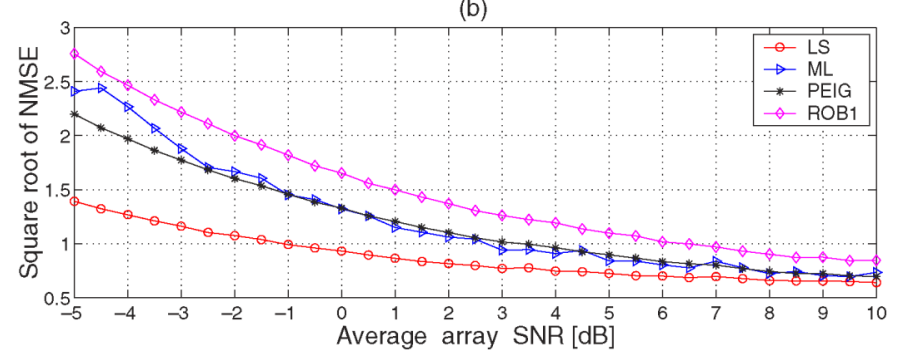

Fig. 1. Square root of the normalized MSE as a function of average array SNR assuming known values of $\mathbf{m}, \mathbf{C}$, and $\mathbf{R}$. (a) Diagonal C. (b) Nondiagonal $\mathbf{C}$.

(a)

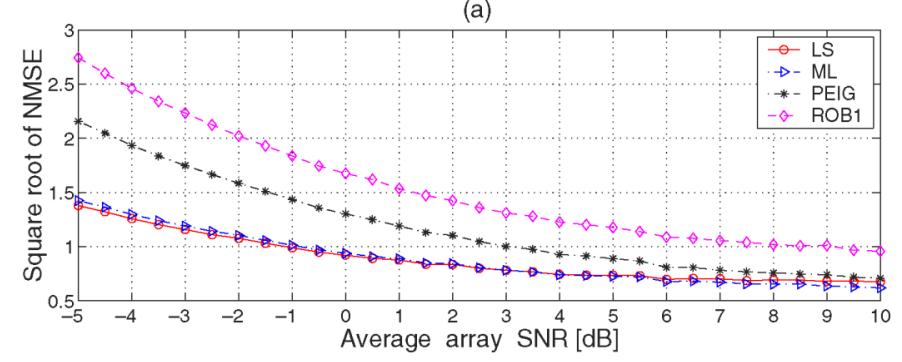

(b)

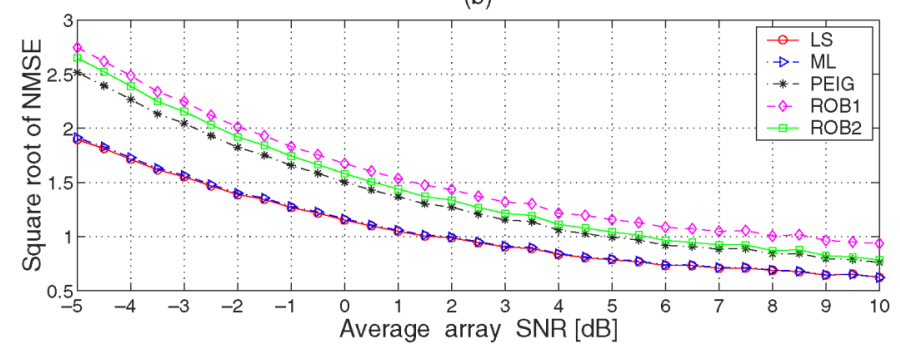

Fig. 2. Square root of the normalized MSE as a function of average array SNR for nondiagonal $\mathbf{C}$. (a) Assuming known $\mathbf{m}$ and $\mathbf{C}$ and using an estimate for R. (b) Assuming known $\mathbf{m}$ and using estimates for $\mathbf{R}$ and $\mathbf{C}$.

such that the expected value over the steering vector error equals to $\epsilon_{1}: \operatorname{Tr}(\mathbf{C})=\epsilon_{1}$, where $\operatorname{Tr}(\mathbf{C})=M \nu$; hence, $\nu=\epsilon_{1} / M$. In this case, we set $\epsilon_{1}=4$ and $\epsilon_{2}=1$. Note that the values of $\epsilon_{1}$ and $\epsilon_{2}$ were chosen such that the ROB1 and ROB2 beamformers have the best average performance for all SNR ranges illustrated. For the ML beamformer, under all assumptions, we chose the initialization vector and its stopping criterion as suggested in [11]. The initialization is performed with the Capon beamformer, and the stopping criterion is $\left\|\mathbf{w}^{(n+1)}-\mathbf{w}^{(n)}\right\| \leq 0.001 / \sqrt{M}$, where $n$ is the iteration number.

In all figures, we illustrate the square root of the NMSE as a function of SNR for UR $=0 \mathrm{~dB}$. Fig. 1(a) and (b) shows the performance using the LS, PEIG, ROB1, and ML beamformers assuming known $\mathbf{m}, \mathbf{C}$, and $\mathbf{R}$ for scenarios 1) and 2), respectively. It can be seen that for scenario 1), the LS beamformer outperforms the other methods for the SNR range between $-5 \mathrm{~dB}$ to $4 \mathrm{~dB}$, whereas for scenario 2), it outperforms the others methods 
(a)

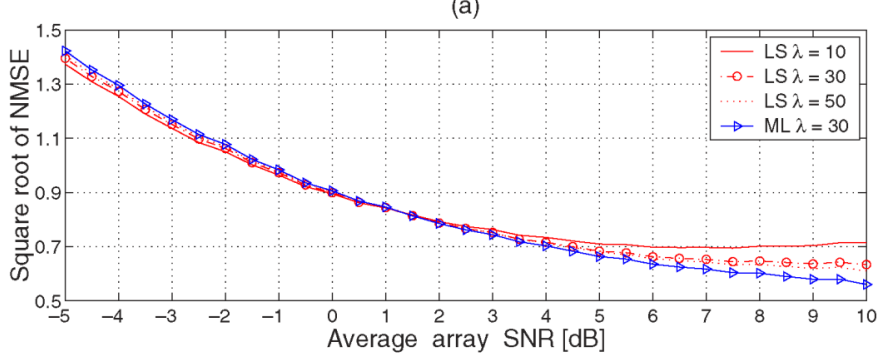

(b)

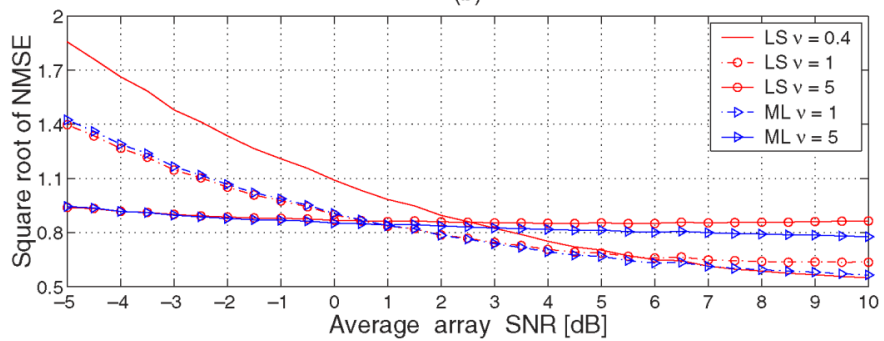

Fig. 3. Square root of the normalized MSE as a function of average array SNR for diagonal $\mathbf{C}$. (a) Assuming known $\mathbf{m}$ and $\mathbf{C}$ and $\mathbf{R}$ estimated with three different values for $\lambda$. (b) Assuming known $\mathbf{m}, \mathbf{R}$ estimated with $\lambda=30$, and C estimated with three different values for $\nu$.

throughout the SNR range illustrated. Note that for scenario 2), the performance of the ML beamformer oscillates in all the SNR ranges. It is observed that for some experiments, the ML algorithm requires a larger number of iterations to achieve its convergence. Additional numerical examples show that for both scenarios, the ML beamformer improves its performance relative to the LS strategy as the values of $M, N$, and UR increase. From (7), the value of the denominator gets larger as $\sigma_{a}^{2}$ or $\sigma_{g}^{2}$ increases its value; hence, for a larger UR, the elements of the LS beamformer vector tend to zero, and the NMSE goes to one. In general, for known $\mathbf{m}, \mathbf{C}$, and $\mathbf{R}$, the LS approach outperforms the other methods mainly for low values of M, SNR, and UR.

Next, we illustrate the performance of the beamformers for known $\mathbf{m}$ and $\mathbf{C}$ and estimated $\mathbf{R}$. Fig. 2(a) illustrates the performance using the LS, PEIG, ROB1, and ML beamformers for scenario 2). It can be seen that the LS and ML approaches have a similar performance for the SNR range between -5 and $8 \mathrm{~dB}$. Additional numerical examples (not shown here) show that beyond $15 \mathrm{~dB}$, the NMSE of PEIG, LS, and ML beamformers degrades approaching one as SNR increases. This is because the signal is continuously present throughout the data used to estimate $\mathbf{R}$. As expected, the use of a diagonal load helps to improve the performance for large SNR. As an example, we illustrate in Fig. 3(a) the NMSE as a function of SNR using the LS and ML beamformers for scenario 1) with $\lambda$ equal to 10 , 30 , and 50. It can be seen that the performance deteriorates for lower $\lambda$ values as SNR increases. Note that we show one curve for the ML method since its performance does not vary much in the SNR range illustrated; however, similar conclusions can be drawn for larger SNR values.

Finally, we illustrate the performance of the beamformers assuming known $\mathbf{m}$ and using estimates for $\mathbf{R}$ and $\mathbf{C}$. Fig. 2(b) shows the NMSE using the LS, PEIG, ROB1, ROB2, and ML beamformers for scenario 2). The LS and ML approaches show similar NMSE in the SNR range illustrated. We also show the performance of LS and ML beamformers for different values of $\nu$. Fig. 3(b) shows the NSME as a function of SNR using the LS and ML beamformer for scenario 1) with $\nu$ equal to $\epsilon_{1} / M=0.4,1$ (current value of $\sigma_{a}^{2}$ ), and 5. It is observed that for larger values of $\nu$, the NMSE improves for negative SNR values; however, as SNR increases, the best performance is obtained for the lowest value of $\nu$.

\section{CONCLUSION}

We proposed a simple, linear beamformer for estimating a signal waveform in the presence of steering vector uncertainties. Our approach is based on an expected LS criterion. We have shown through numerical examples the robustness of our method in terms of the NMSE for random steering vectors mainly for low SNR and UR values. The simple implementation, closed-form solution, and good performance establish this method as an attractive alternative compared with the previously proposed ROB1, ROB2, ML, and PEIG beamformers.

\section{REFERENCES}

[1] B. D. van Veen and K. M. Buckley, "Beamforming: A versatile approach to spatial filtering," IEEE Signal Process. Mag., vol. 5, no. 2, pp. 4-24 Apr. 1988.

[2] J. Yang and A. Swindlehurst, "Signal copy with array calibration errors," in Proc. 27th Asilomar Conf. Signals, Systems, Computers, vol. 2, Nov. 1-3, 1993, pp. 405-413.

[3] S. A. Vorobyov, A. B. Gershman, and Z.-Q. Luo, "Robust adaptive beamforming using worst case performance optimization," IEEE Trans. Signal Process., vol. 51, no. 2, pp. 313-324, Feb. 2003.

[4] B. Friendlander and A. Weiss, "Direction finding in the presence of mutual coupling," IEEE Trans. Antennas Propag., vol. 39, no. 3, pp. 273-284, Mar. 1991

[5] J. Li, P. Stoica, and Z. Wang, "On robust capon beamforming and diagonal loading," IEEE Trans. Signal Process., vol. 51, no. 7, pp. 1702-1715, Jul. 2003.

[6] K. L. Bell, Y. Ephraim, and H. L. van Trees, "A Bayesian approach to robust adaptive beamforming," IEEE Trans. Signal Process., vol. 48, no. 2 , pp. 386-398, Feb. 2000.

[7] H. Cox, "Effects of random steering vector errors in the Applebaum array,” J. Acoust. Soc. Amer., vol. 54, pp. 771-785, Sep. 1973.

[8] A. Paulraj and T. Kailath, "Direction of arrival estimation by eigenstructure methods with imperfect spatial coherence of wave fronts," J. Acoust. Soc. Amer., vol. 83, pp. 1034-1040, Mar. 1988.

[9] A. B. Gershman, C. F. Mecklenbräuker, and J. F. Böhme, "Matrix fitting approach to direction of arrival estimation with imperfect spatial coherence of wavefronts," IEEE Trans. Signal Process., vol. 45, no. 7, pp. 1894-1899, Jul. 1997.

[10] S. Shahbazpanahi, A. B. Gershman, Z.-Q. Luo, and K. M. Wong, "Robust adaptive beamforming for general-rank signal models," IEEE Trans. Signal Process., vol. 51, no. 9, pp. 2257-2269, Sep. 2003.

[11] O. Besson, A. A. Monakov, and C. Chalus, "Signal waveform estimation in the presence of uncertainties about the steering vector," IEEE Trans. Signal Process., vol. 52, no. 9, pp. 2432-2439, Sep. 2004.

[12] A. B. Gershman, "Robust adaptive beamforming in sensor arrays," Int J. Electron. Commun., vol. 53, pp. 305-324, Dec. 1999

[13] Y.C. Eldar and A. Nehorai, "Mean-squared error beamforming for signal estimation: A competitive approach," in Robust Adaptive Beamforming, J. Li and P. Stoica, Eds. Hoboken, NJ: Wiley, 2006, pp. 259-298.

[14] Y. C. Eldar, "Minimax estimation of deterministic parameters in linear models with a random model matrix," IEEE Trans. Signal Process., vol. 54, no. 2, pp. 601-612, Feb. 2006.

[15] W. James and C. Stein, "Estimation of quadratic loss," in Proc. 4th Berkeley Symp. Math. Stat. Prob., vol. 1, Berkeley, CA, 1961, pp. 361-379.

[16] W. E. Strawderman, "Proper Bayes minimax estimators of multivariate normal mean," Ann. Math. Stat., vol. 42, pp. 385-388, 1971.

[17] Z. Ben-Haim and Y. C. Eldar, "Blind minimax estimators: Improving on least squares estimation," in IEEE Workshop Statistical Signal Processing (SSP'05), Bordeaux, France, Jul. 17-20, 2005.

[18] O. Besson and F. Vincent, "Performance analysis of beamformers using generalized loading of the covariance matrix in the presence of random steering vector errors," IEEE Trans. Signal Process., vol. 52, no. 2, pp. 452-459, Feb. 2005 